\title{
Molecular Testing of Thyroid Nodules: Distinguishing Misuse from Appropriate Use
}

\author{
Linwah Yip, MD and Sally E. Carty, MD \\ Division of Endocrine Surgery and Surgical Oncology, Department of Surgery, University of Pittsburgh, Pittsburgh, PA
}

Diagnostic surgery or repeat biopsy is currently indicated for the $\sim 25 \%$ of nodules with fine-needle aspiration biopsy results that are cytologically indeterminate [i.e., classified according to the Bethesda System for Reporting Thyroid Cytopathology as atypia of undetermined significance/follicular lesion of undetermined significance (AUS/ FLUS), follicular neoplasm or suspicious for follicular neoplasm, or suspicious for malignancy]. ${ }^{1}$ The dual goals of preoperative evaluation are to limit unnecessary thyroid surgery and perform the correct procedure in one initial step, if possible. Using molecular profiling as a diagnostic adjunct to improve risk stratification of cytologically indeterminate thyroid nodules has been the topic of active investigation. Studying the clinical effectiveness of integrating molecular tests into practice is much needed and was the focus of the article by Han et al., "The Impact of Molecular Testing on the Surgical Management of Patients with Thyroid Nodules."

To date, the two commonly used methodologies are the gene expression classifier (GEC) and somatic mutation testing (MT), which are used for divergent clinical goals. GEC relies on the expression pattern of a panel of 147 genes specifically selected to optimize the negative predictive value (NPV), and in a multiinstitutional study of 265 indeterminate nodules, test sensitivity and specificity were 92 and $52 \%$, respectively. ${ }^{2}$ The high sensitivity associated with GEC may improve identification of nodules with low

This is an editorial note on the title available at doi:10.1245/s10434014-3508- $\mathrm{x}$.

(C) Society of Surgical Oncology 2014

First Received: 20 January 2014;

Published Online: 7 February 2014

S. E. Carty, MD

e-mail: cartyse@upmc.edu malignancy risk and select those nodules that may not need a histologic diagnosis. However, the modest specificity does not reliably identify those nodules with a high risk of malignancy; thus, positive GEC results should not be used to guide extent of initial thyroidectomy. In contrast, MT evaluates for somatic DNA mutations that are known to be involved in thyroid carcinogenesis and for cytologically indeterminate nodules, a comprehensive MT panel that includes point mutations in $B R A F$ and $R A S$, as well as gene rearrangements such as RET/PTC1 or 3 , and $P A X 8 / P P A R \gamma$ provides test sensitivity and specificity of 61 and $98 \%$, respectively. ${ }^{3}$ Because MT can identify nodules with a high malignancy risk, it can productively be used to direct extent of the thyroidectomy in real time. ${ }^{4}$ A negative MT result does not currently eliminate the risk of cancer; thus, thyroidectomy is still indicated. A study design that compares the clinical efficacy of these available molecular tests using the same end point is therefore inaccurate and does not account for each test's strengths and indications for use.

In the study by Han et al., a series of 87 patients with cytology, molecular testing, and histology were studied to determine whether molecular profiling directed the appropriate initial surgery. However, only 40 patients had indeterminate cytology, whereas almost half of the study cohort had molecular tests for either benign or malignant cytology results. There is currently no clinical indication for obtaining GEC for cytologically benign nodules or MT for cytologically malignant nodules. Furthermore, outside of an investigative protocol, no data support obtaining both sets of molecular tests, particularly for nodules that are already cytologically malignant which was done for two patients in this series. We agree with the authors' conclusion that molecular profiling may indeed be inappropriately used in patients for whom the results would not alter clinical management, i.e., those with benign or malignant cytology, and echo their words of caution when considering who should have this testing performed. 
Among the 40 patients with indeterminate cytology, which was the subset for whom any molecular profiling was indicated, 22 patients had GEC, and 18 had somatic molecular testing in some form. Only 1 patient with GEC negative results had surgery, and histology was benign (NPV $100 \%$ ), yet no long-term follow-up data were presented to exclude missed malignancy among those who did not have thyroidectomy. Among the 18 patients with somatic testing, an incomplete panel (BRAF, NRAS, and $R E T / P T C$ alone) was used for seven patients, and the actual comprehensive MT panel (BRAF, N/K/H-RAS, RET/PTC, and $P A X 8 / P P A R \gamma)$ was used for 11 patients; 6 positive results were identified, and all had histologic malignancy [positive predictive value (PPV), $100 \%$ ]. The molecular tests were optimized for nodules with indeterminate cytology, and the observed NPV for GEC and PPV for MT were consistent with prior studies. However, the incidence of malignancy in the 40 indeterminate nodules was rather high, at $57 \%$. In addition, in the typically low-risk AUS/ FLUS cohort, the literature-reported malignancy risk is 15-25\%, yet this study cohort had an associated malignancy risk that was approximately twofold higher $(47 \%))^{2,3,5}$ Because cancer prevalence affects NPV and PPV, but does not affect sensitivity and specificity, the adaptation of molecular profiling results into management algorithms should be considered after careful review of institution and regional variances in malignancy rates associated with each cytology category.

The management algorithm used as the measure of appropriate surgery by Han et al. was inclusive of symptomatology and presence of thyroid dysfunction and was not implemented prospectively. Although clinical considerations certainly contribute to deciding the extent of initial thyroidectomy, these are sometimes difficult to elucidate retrospectively and, furthermore, are not parameters that necessarily affect malignancy risk or molecular testing results. Because fine-needle aspiration biopsy results predict cancer with less than complete accuracy, any determination of the appropriate initial extent of surgery for cancer must include either histology or long-term follow-up. In another cohort study evaluating how MT use optimizes the initial extent of thyroidectomy, histology was the gold standard for surgery performed per the American
Thyroid Association evidence-based guidelines, and total thyroidectomy for histologic thyroid cancer $\geq 1 \mathrm{~cm}$ or thyroid lobectomy for microcarcinoma or benign disease were considered the appropriate extent of initial surgery. ${ }^{6}$ Routine MT use in nodules that were cytologically AUS/ FLUS or follicular neoplasm reduced the need for 2-stage thyroidectomy by 2.5 -fold, and MT beneficially improved preoperative decision-making when used systematically and appropriately. ${ }^{4}$

Some of the pitfalls of incorporating new technology into routine clinical practice are highlighted in this study. In any multidisciplinary specialty, there is potential for inappropriate use of tests that are thought to help facilitate the decision-making for the next physician in the consultative chain. However, the interpretation of test results to guide surgical management is, in the end, up to the surgeon. An objective evaluation of published data and an understanding of how performance parameters apply to specific clinical scenarios are essential before the information is used to make decisions about patient care.

\section{REFERENCES}

1. Baloch ZW, LiVolsi VA, Asa SL, et al. Diagnostic terminology and morphologic criteria for cytologic diagnosis of thyroid lesions: a synopsis of the National Cancer Institute Thyroid Fine-Needle Aspiration State of the Science Conference. Diagn Cytopathol. 2008;36:425-37.

2. Alexander EK, Kennedy GC, Baloch ZW, et al. Preoperative diagnosis of benign thyroid nodules with indeterminate cytology. $N$ Engl J Med. 2012;367:705-15.

3. Nikiforov YE, Ohori NP, Hodak SP, et al. Impact of mutational testing on the diagnosis and management of patients with cytologically indeterminate thyroid nodules: a prospective analysis of 1056 FNA samples. J Clin Endocrinol Metab. 2011;96:3390-7.

4. Yip L WL, Armstrong MJ, Silbermann A, McCoy KL, Stang MT, et al. A clinical algorithm for fine-needle aspiration molecular testing effectively guides the appropriate extent of initial thyroidectomy. Ann Surg. In press.

5. Bongiovanni M, Spitale A, Faquin WC, et al. The Bethesda System for Reporting Thyroid Cytopathology: a meta-analysis. Acta Cytol. 2012;56:333-9.

6. American Thyroid Association (ATA) Guidelines Taskforce on Thyroid Nodules and Differentiated Thyroid Cancer, Cooper DS, Doherty GM, et al. Revised American Thyroid Association management guidelines for patients with thyroid nodules and differentiated thyroid cancer. Thyroid. 2009;19:1167-214. 